\title{
Pretreatment with antioxidants prevent bone injury by improving bone marrow microenvironment for stem cells
}

\author{
Lingling Xian ${ }^{1,2}$, Michael Lou ${ }^{1}$, Xiangwei $\mathrm{Wu}^{1,2}$, Bing $\mathrm{Yu}^{1}$, Frank Frassica ${ }^{1}$, Mei Wan ${ }^{1}$, \\ Lijuan Pang ${ }^{1,2}$, Chunyi Wen $^{3}$, Erik Tryggestad ${ }^{4}$, John Wong $^{4}, \mathrm{Xu} \mathrm{Cao}^{{ }^{*}}$ \\ ${ }^{1}$ Department of Orthopaedic Surgery, School of Medicine, Johns Hopkins University, Baltimore, USA; \\ *Corresponding Author: xcao11@jhmi.edu \\ ${ }^{2}$ Shihezi Medical College, Shihezi University, Shihezi, China \\ ${ }^{3}$ Department of Orthopaedics, University of Hong Kong, Hong Kong, China \\ ${ }^{4}$ Radiation Oncology Medical Physics, School of Medicine, Johns Hopkins University, Baltimore, USA
}

Received 15 April 2012; revised 12 May 2012; accepted 11 June 2012

\begin{abstract}
Irradiation induces bone injury by generating free radicals that adversely affect the microenvironment for Mesenchymal stem cells (MSCs) and damages bone marrow blood vessels. We wished to investigate the efficacy of antioxidant administration in protecting stem cell microenvironments and promoting bone marrow vasculature recovery after radiation treatment. The antioxidant ascorbic acid was administered 3 times at a dosage: $150 \mathrm{mg} / \mathrm{kg} /$ day to experimenttal groups 3 days before targeted radiation by a unique Small Animal Radiation Research Platform (SARRP). Histological staining indicated that antioxidant treated mice had less severe bone marrow damage 1 week after irradiation with substantial marrow cellular recovery 4 weeks later. Flow cytometry analysis showed that antioxidant administration was correlated with a rebound in MSC quantity in bone marrow. Anti-oxidant treatment was also observed to allow for better vasculature retention and recovery through angiographic imaging. Our data suggests that pre-treatment with ascorbic acid serves to improve bone marrow microenvironments for bone marrow stem cells after radiation treatment.
\end{abstract}

Keywords: Irradiation; Bone Injury; Stem Cells; Microenvironment; Antioxidant

\section{INTRODUCTION}

Radiation therapy has been widely utilized in the clinical setting to treat a variety of malignancies such as gliomas of the brain, prostate tumors, lung carcinomas, lymphoma, gynecologic cancers, and breast cancers [1-3]. Ionizing radiation damages bone tissue resulting in bone density loss and an increased risk of fractures [4,5]. Bone insufficiency can be as high as $45 \%$ amongst female patients post-radiation treatments for cervical cancers [6]. Animal models suggest substantial loss of trabecular bone after irradiation [7]. Currently, few preventive steps are taken to ensure maximized recovery after radiation treatment.

Ionizing radiation creates oxidative stress by generating free radicals and reactive oxygen species that damage major cellular components such as proteins, membranes, and nucleic acids [8]. Hematopoietic stem cell sensitivity to radiation is well documented [9]. However, the response and sensitivity of MSCs to radiation is not well studied. MSCs differentiation fates include osteogenic and adipogenic lineages, hence their involvement in bone remodeling and repair $[1,10]$. Bone marrow cells experience high sensitivity to the effects of ionizing radiation as demonstrated in murine animal models [11]. Irradiation creates oxidative cellular damage, along with resulting in marrow microenvironment changes weeks after administration [1]. Therefore, curbing the pervasiveness of damage from free radicals would be an effective strategy for clinical recovery post-treatment.

Damaging free radicals arising from irradiation in the bone microenvironment affects bone marrow stem cell viability [1]. Damage to bone marrow vasculatures, major stem cell niches, likely affects the recovery of MSCs in the irradiated areas. In this study, we investigated the effects of antioxidant administration on bone marrow stem cells and residing microenvironment after irradiation. The Small Animal Research Radiation Platform (SARRP) we utilized allows for precise radiation administration with resolution up to $1 \mathrm{~mm}$ [12]. Our find- 
ings suggest that administration of the antioxidant ascorbic acid lessened the extent of radiation damage of bone marrow micro-environments for stem cells, and improves recovery prognosis.

\section{MATERIALS AND METHODS}

\subsection{Mice}

8-week-old c57BL/6 male mice were given per os (p.o.) ascorbic acid at doses of 0 , and $150 \mathrm{mg} / \mathrm{kg} /$ day 3 days before irradiation. The irradiation procedure was done as previously reported [1]. Briefly, a clinically applicable dose rate of (4 Gy $\mathrm{min}^{-1}$ ) was delivered using $225 \mathrm{kVp}$ with a $3.0 \mathrm{~mm}$ focal spot with up to a $13 \mathrm{~mA}$ beam current. Irradiation was given once per day for 5 days, wit the total dose at 20 Gys. All animals were maintained in the animal facility of the Johns Hopkins University School of Medicine. The experimental protocol was reviewed and approved by the Institutional Animal Care and Use Committee of the Johns Hopkins University, Baltimore MD, USA.

\subsection{Histochemistry and Histomorphometric Analysis}

At the time of sacrifice, proximal and distal femora of mice 1 week and 4 weeks post-irradiation treated or untreated with ascorbic acid were resected and fixed in $10 \%$ buffered formalin for 48 hours, decalcified in $10 \%$ ethylenediamine tetraacetic acid (EDTA) (pH 7.4) for 20 days, and embedded in paraffin. 4- $\mu$ m-thick longitudenally oriented sections of bone including the metaphysis and diaphysis were processed for hematoxylin and eosin (H\&E) staining. Sections were microphotographed to perform histomorphometric measurements on the highlighted areas of the bone displayed on the digitalized image. Quantitative histomorphometric analysis was conducted in a blinded fashion with OsteoMeasure XP Software (OsteoMetrics, Inc., Decatur, GA, USA). 2-dimensional parameters of trabecular bone were measured in a $2 \mathrm{~mm}$ square, one-mm distal to the lowest point of the growth plate in the trabecular bone.

\subsection{CFU-F and CFU-Ob Assays}

At the time of sacrifice, the femurs were cut in half, into proximal and distal pieces, and bone marrow from medullary cavities were collected individually. Cell number was determined after removal of red blood cells with Zapoglobin (Coulter Corp.). For assays of CFU-F and CFU-Ob number, $1 \times 10^{6}$ murine marrow cells were plated into six well plates in $2 \mathrm{~mL} \alpha$-MEM supplemented with glutamine (2 $\mathrm{mM} \mathrm{az})$, penicillin $(100 \mathrm{U} / \mathrm{mL})$, streptomycin sulfate $(100 \mu \mathrm{g} / \mathrm{mL})$, and $20 \%$ lot-selected FBS. Duplicate cultures were established. After 2 - $3 \mathrm{hr}$ of adhesion, unattached cells were removed, and $2.5 \times 10^{6}$ irradiated guinea pig feeder cells (provided by Dr. Bren-dan J. Canning) were added to the cultivation medium of adherent cultures immediately after washing. On day 14 , cultures were fixed and stained with $0.5 \%$ crystal violet. Colonies containing 50 or more cells were noted. For CFU-Ob assay, the cells were cultured with osteogenic medium $\left(10 \% \mathrm{FBS}, 10^{-7} \mathrm{M}\right.$ dexamethasone, 10 $\mathrm{mM} \beta$-glycerol phosphate and $50 \mu \mathrm{M}$ ascorbate-2-phos phate) for 21 days and analyzed with alizarin red staining. The colony-forming efficiency was determined by number of colonies per $10^{6}$ marrow cells plated.

\subsection{Fluorescence-Activated Cell Sorting (FACS) Analysis}

Cell aliquots isolated from bone marrow were incubated for 20 minutes at $4^{\circ} \mathrm{C}$ with phycoerythrin (PE)-, fluorescein isothiocyanate (FITC)-, peridinin chlorophyll protein (Per CP)-, and allophycocyanin (APC)-conjugated antibodies against mouse Sca-1, CD29, CD45, and CD11b (Bio-legend). Acquisition was performed on a fluorescence-activated cell sorting (FACS) Aria model (BD Biosciences), and analysis was performed using a FACS DIVE software version 6.1.3 (BD Biosciences).

\subsection{Measurement of Lipid Oxidation}

Peripheral venous blood was drawn into syringes containing preservative-free heparin $(25 \mathrm{U} / \mathrm{mL}$, Gibco, Life Technologies, Gaithersburg, MD) and centrifuged at $3500 \mathrm{rpm}$ for 10 minutes at $5^{\circ} \mathrm{C}-10^{\circ} \mathrm{C}$ to isolate the plasma. Bone marrow samples were collected into preservative-free heparinized saline $(25 \mathrm{U} / \mathrm{mL})$. And centrifuged at $3500 \mathrm{rpm}$ for $10 \mathrm{~min}$ at $5^{\circ} \mathrm{C}-10^{\circ} \mathrm{C}$ to isolate bone marrow plasma. Plasma lipid oxidation was assessed by determining the level of Thiobarbituric Acid Reactive Substances (TBARS) using a TBARS assay kit (zep-tometrix).

\subsection{Micro-CT Angiography Analysis}

Blood vessels in long bone were imaged by contrast-enhanced micro-CT angiography. The thoracic cavity was opened after the animals were anesthetized. The vasculature was flushed with $0.9 \%$ normal saline solution containing heparin sodium $(100 \mathrm{U} / \mathrm{mL})$ through a needle inserted into the left ventricle, and then pressure fixed with $10 \%$ neutral buffered formalin. After that, the vessels were flushed again using heparinized saline solution. Finally, the vasculature was perfused with a radiopaque silicone rubber compound containing lead chromate (Microfil MV-122; Flow Tech). The corpses were stored at $4^{\circ} \mathrm{C}$ overnight for contrast agent polymerization. Mouse femurs were dissected from the bodies and soaked for 4 days in 10\% neutral buffered formalin to ensure complete tissue fixation. Specimens were subsequently treated for 48 hours in a formic acid-based 
solution (Cal-Ex II) for decalcification in order to visualize the vasculature inside bone. Images were obtained using a high-resolution micro-CT imaging system $(5.7-\mu \mathrm{m}$ isotropic voxel size) (Skyscan 1172, Kontich, Belgium). Isotropic voxel size for the scans is $5.5 \mu \mathrm{m}$. X-ray voltage of $50 \mathrm{kV}$ and $200 \mu \mathrm{A}$ were applied for image acquisition. After standardized reconstruction by a modified Feldkamp algorithm via SkyScan recon software, the datasets were analyzed using SkyScan CT-analyser software. The vasculature was then examined with $\mathrm{ANT}^{\mathrm{TM}}$ software (SkyScan) by the following parameters: Vessel volume/tissue volume, vessel number and vessel thickness.

\subsection{Statistics}

Data were presented as the mean \pm SEM and analyzed using an one-way analysis of variance (ANOVA) fol- lowed by Dunnett's test.

\section{RESULTS}

\subsection{Effects of Antioxidant Administration on Bone Marrow Microenvironment after Local Irradiation}

The SARRP device was utilized to provide an accurate delivery of radiation at $1 \mathrm{~mm}$ resolution with additional aid provided by micro-CT and X-ray imaging. C57BL/6 mice were irradiated at the left distal (LD) half of the femur (Figure 1(a)) with a dosage of 4 Grays once a day for 5 consecutive days with the cumulative amount of radiation exposure at 20 Grays. In order to examine the effects of anti-oxidant administration on MSCs and vascular changes post-irradiation, the antioxidant ascorbic acid or Vitamin $\mathrm{C}$ was orally given at $150 \mathrm{mg} / \mathrm{kg} /$ day 3

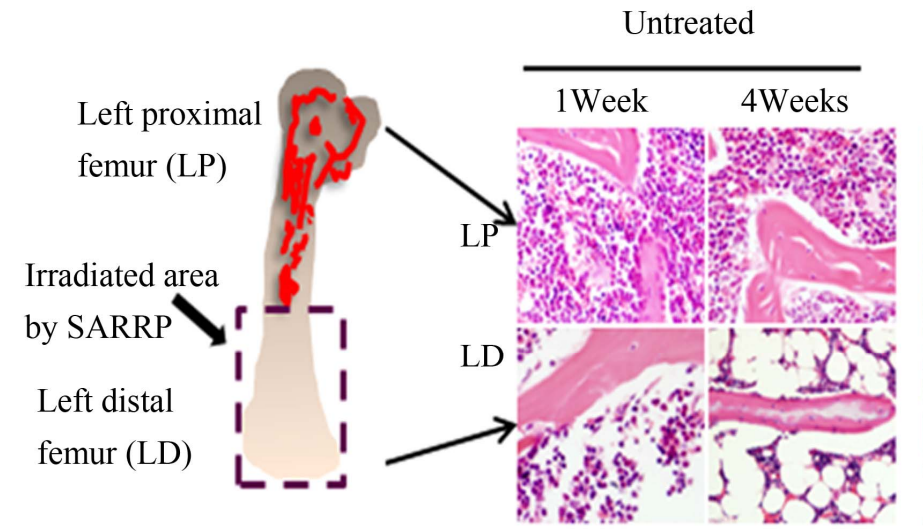

(a)

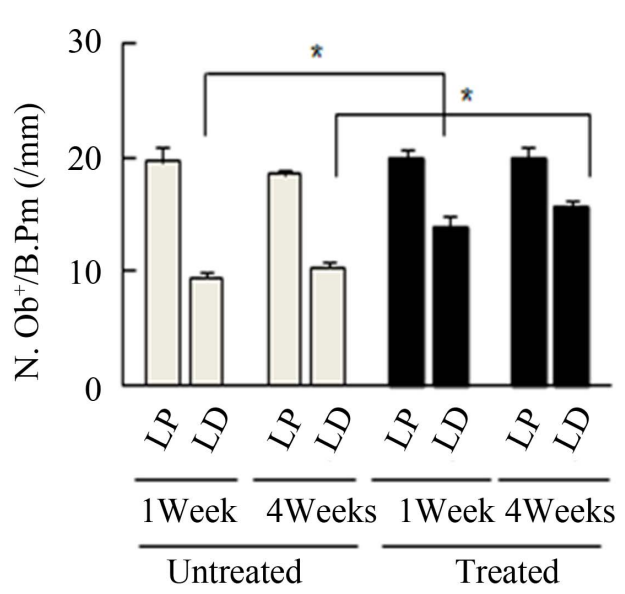

(c)

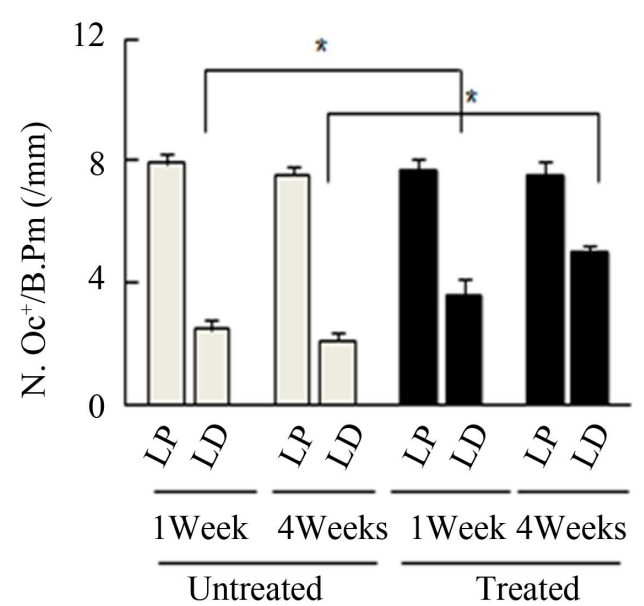

(d)

Figure 1. H\&E-staining and bone histomorphometric analysis of trabecular bone from the ascorbic acid treated or untreated c57BL/6 male mice 1 week and 4 weeks post-local irradiation; (a) Model of local irradiation on left distal femur; (b) Light micrographs of H\&E-staining on trabecular bone sections from proximal and distal femora of mice 1 week and 4 weeks post-irradiation treated or untreated with ascorbic acid. LP: Left proximal femur, LD: Left distal femur, Scale bar: $50 \mu \mathrm{m}$; (c) Number of osteoblasts per bone perimeter $\left(\mathrm{N} . \mathrm{Ob}^{+} / \mathrm{B} . \mathrm{Pm}\right)$; (d) Number of osteoclasts per bone perimeter (N.Oc $\left.{ }^{+} / \mathrm{B} . \mathrm{Pm}\right)$. Data represent the mean \pm SEM; $\mathrm{n}=10 ;{ }^{*} p<0.05$. 
days before irradiation. The control group received no ascorbic acid. The left proximal (LP) femur area did not receive direct radiation. Histological staining indicated that the amount of radiation damage was extensive in the LD femoral areas at 1 week and this damage was also found at 4 weeks after radiation treatment (Figure 1(b)). Significant bone marrow cell ablation and irradiation induced adipogenesis can be observed in the LD antioxidant untreated mice even 4 weeks after radiation. The LD antioxidant treated mice had less severe damage 1 week after irradiation with substantial marrow cellular recovery at 4 weeks (Figure 1(b)). The results suggest that irradiation damages the bone marrow microenvironment and antioxidant treatment may be able to lessen the extent of that damage.

We analyzed the effects of irradiation and antioxidant treatment on bone cells with histomorphometry. Our results indicated that osteoblast and osteoclast cell number were significantly lower in the left distal femoral sections (LD) 1 and 4 weeks after irradiation when compared with proximal femoral controls (LP) (Figures 1(c) and (d)). The mice showed increased numbers of osteoclast and osteoblast cells when treated with the antioxidant in 1 week and 4 weeks post-treatment in comparison to their untreated counterparts (Figures 1(c) and (d)). The results support that treatment with antioxidants elevated the level of bone cells 1 week and 4 weeks after irradiation.

\subsection{Effects of Antioxidant Administration on MSCs Potential Post-Irradiation}

Bone Marrow Mesenchymal Stem Cells (BMSCs) were isolated from the Right Distal (RD), Right Proximal (RP), Left Distal (LD), and Left Proximal (LP) quadrants. A CFU-Fibroblast (CFU-F) assay was then conducted to examine the effects of irradiation on BMSCs. In the antioxidant untreated left femur isolates (LD and LP), none to very few numbers of cells were colony forming even 4 weeks after irradiation. However, in the antioxidant treated isolates, viable colony forming cells were observed at 1 and 4 weeks post irradiation. CFU-F counts in antioxidant treated LD and LP isolates were significantly higher than their untreated counterparts (Figures 2(a) and (c)). We performed a CFU-Osteoblast (CFU-Ob) assay visualized via alizarin red staining to assess the differentiation potential of BMSC isolates. With antioxidant treatment, differentiated cells obtained from both LD and LP isolates have significantly elevated CFU-Ob counts (Figures 2(b) and (d)). Of note, LP cell numbers are affected despite that quadrant not having gone through direct radiation exposure. The assays suggest that radiation damage is extensive; affecting BMSCs away from the local site of radiation and antioxidant administration appears to improve the number and also the differentiation potential of BMSCs in bone marrow 1 and 4 weeks postradiation.

Previous studies have established that $\mathrm{CD}_{2} 9^{+} \mathrm{Sca}^{+}$

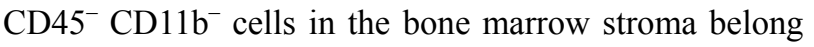
to a subset of MSCs in circulation [13]. Local detection of $\mathrm{CD}_{29}{ }^{+} \mathrm{Sca}^{+} \mathrm{CD}^{+} 5^{-} \mathrm{CD}_{11 b^{-}}$MSCs isolated from irradiated mice showed a very significant drop in MSCs from LD antioxidant treated, untreated and LP treated and untreated 1 week after irradiation (Figure 2(e)). However, in LP antioxidant treated groups 4 weeks post irradiation, MSC numbers have recovered substantially to levels matching our contralateral controls-RD, RP (Figure 2(e)). A similar increase in cell number was also observed for LD antioxidant treated mice as well. Overall, antioxidant treated groups in the LP and LD allowed for significantly higher MSC counts than untreated groups. These results support that administration of ascorbic acid allowed for increased MSC numbers locally following irradiation.

\subsection{Antioxidants Reduce Free Radical Levels from Local Irradiation}

Oxidative stress from radiation damage was monitored utilizing a thiobarbituric acid reactive substances assay (TBARS) with bone marrow isolates from antioxidant treated, and untreated subsets with all four quadrants (LD, LP, RD, RP) present. TBARS assessed the consequences of lipid peroxidation and malondialdehyde levels specifically [1]. In antioxidant untreated mice, LD TBARS levels remained high and relatively unchanged from 1 and 4 weeks after irradiation (Figure 3(a)). In the not directly irradiated LP area, TBARS levels were increased at 1 week and decreased to the basal level at 4 weeks indicating that free radicals diffused ipsilaterally to the proximal femur resulting in MSCs damage. Noticeably, LP antioxidant treated mice had no significant elevation in TBARS levels even 1 week after irradiation. Similarly, LD antioxidant treated mice showed significantly decreased TBARs levels compared to LD untreated mice (Figure 3(a)). TBARS analysis conducted with peripheral blood plasma showed higher levels 1 week after radiation in circulation in the antioxidant untreated group. Antioxidant treated mice demonstrated little to no increase of TBARS in blood plasma after irradiation (Figure 3(b)). Our results demonstrate that irradiation generates free radicals, whose levels decrease after antioxidants are administered.

\subsection{Antioxidant Treatment on MSC Vascular Niche Preservation}

Bone vasculature is a crucial component in the formation and remodeling of bone. Micro-phil perfused femurs 


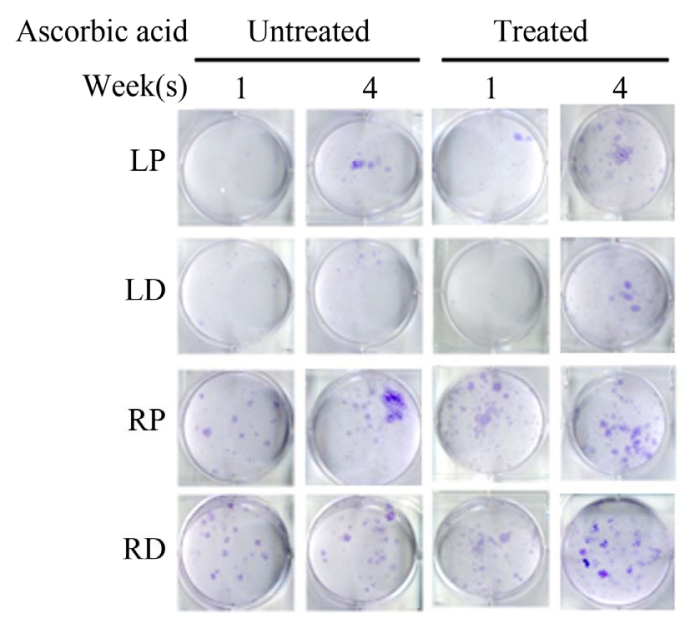

(a)

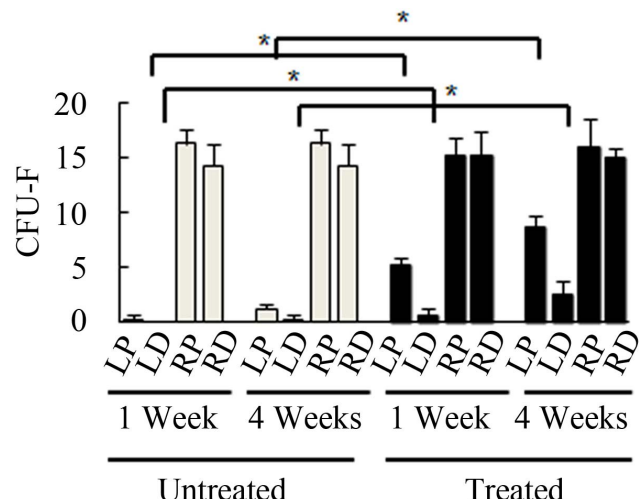

(c)

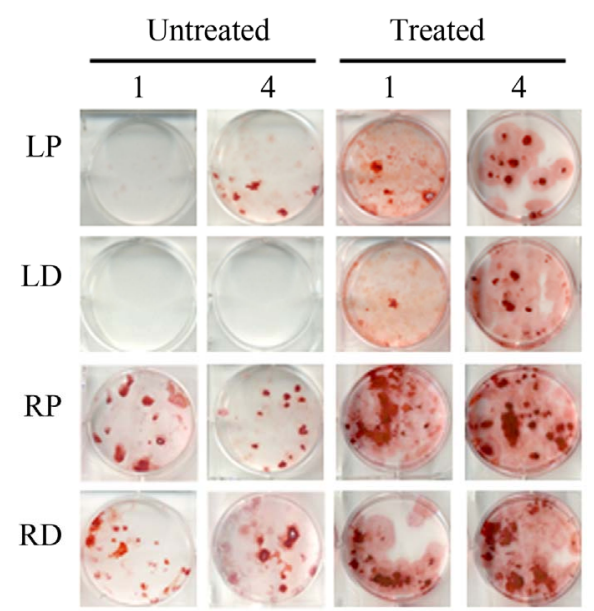

(b)

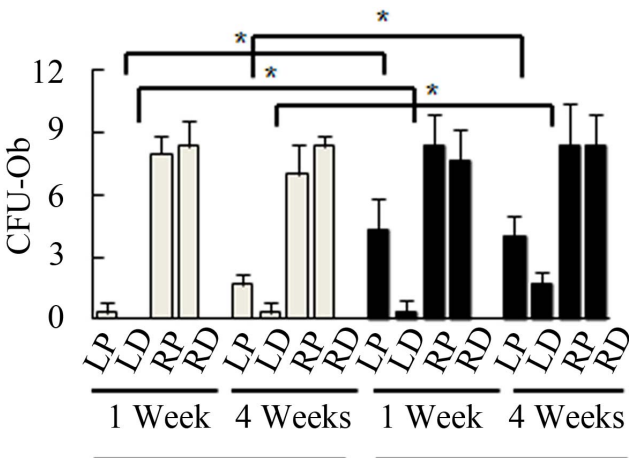

Untreated

(d)

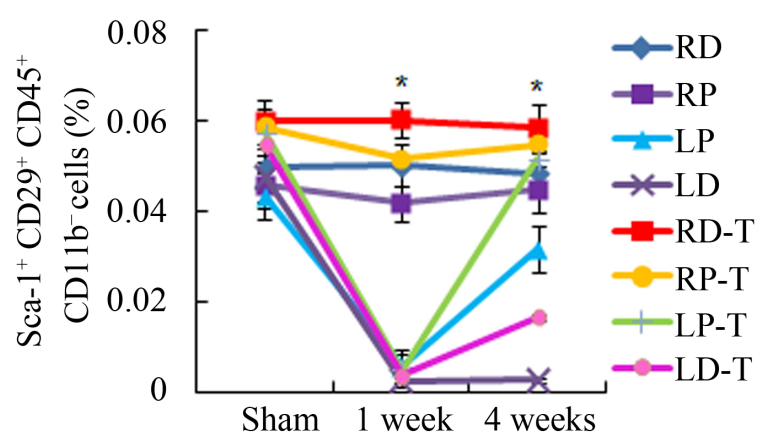

(e)

Figure 2. Antioxidant effects on irradiation induced mesenchymal stem cells change in bone marrow. (a) (b) Colonies formed from harvested femur bone marrow of mice as indicated in CFU-F and CFU-Ob assays (1 $\times 10^{5}$ bone marrow nucleated cells were plated into six-well plates); (c) (d) The colony-forming efficiency was determined by number of colonies per $10^{5}$ marrow cells plated. Data represent the mean \pm SEM of triplicate cultures of bone marrow nucleated cells from five individual mice, ${ }^{*} p<0.05$; (e) FACS analysis of sorted Sca- $1^{+}, \mathrm{CD} 29^{+}, \mathrm{CD}_{4}^{-}$and $\mathrm{CD} 11 \mathrm{~b}^{-}$cells from bone marrow cell suspension obtained from mice. LP: Left proximal femur; LD: Left distal femur; RP: Right proximal femur; RD: Right distal femur. Data represent the mean $\pm \mathrm{SEM} ; \mathrm{n}=10 ;{ }^{*} p<0.05$.

were imaged utilizing 3-dimensional micro-CT and analyzed for parameters after irradiation. 1 week postirradiation, LD sites showed almost complete vasculature ablation (Figure 4(a)). However, in the antioxidant treated mice, there was more intact vasculature compared to the untreated mice in the left femur as a whole (Figure 4(a)). Morphometric results assessing vessel volume, thickness, and number yielded similar results suggesting that mice treated with antioxidants typically had more intact vasculature and suggested more vasculature re- 


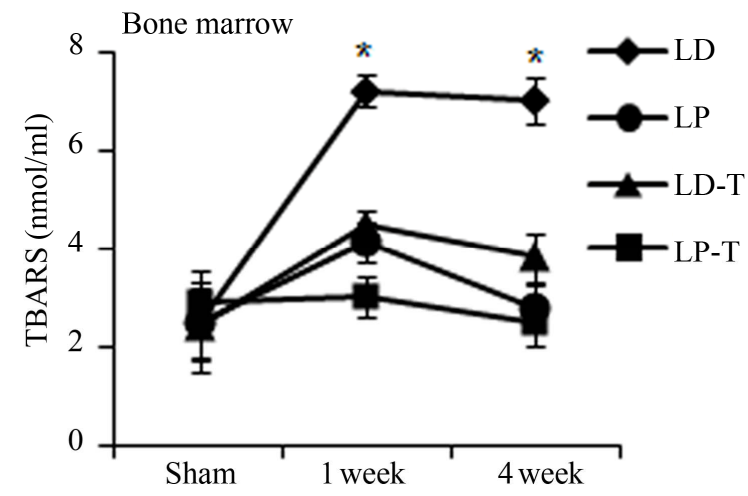

(a)

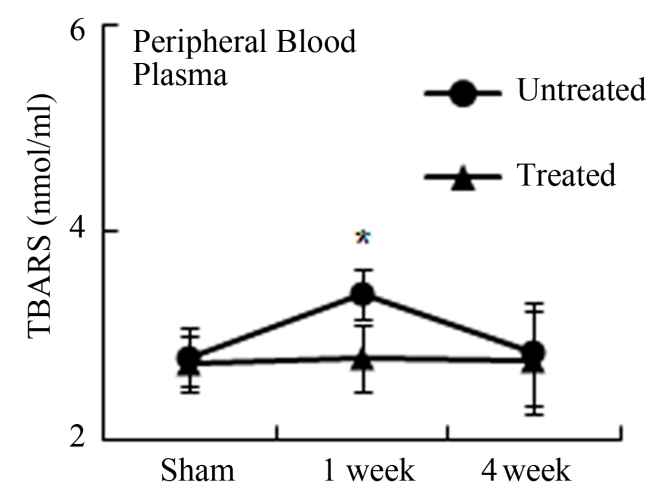

(b)

Figure 3. Antioxidant prevent the production of free radicals by local irradiation. Free radicals were assessed in both bone marrow (a) and peripheral blood (b); Pre-irradiation, and 1 week and 4 weeks post-irradiation detected by thiobarbituric acid reactive substances (TBARS) assay. LP: Left proximal femur; LD: Left distal femur; LP-T: Left proximal femur from mice treated with ascorbic acid; LD-T: Left distal femur from mice treated with ascorbic acid. Data represent the mean \pm SEM; $\mathrm{n}=10 ;{ }^{*} p<0.05$.

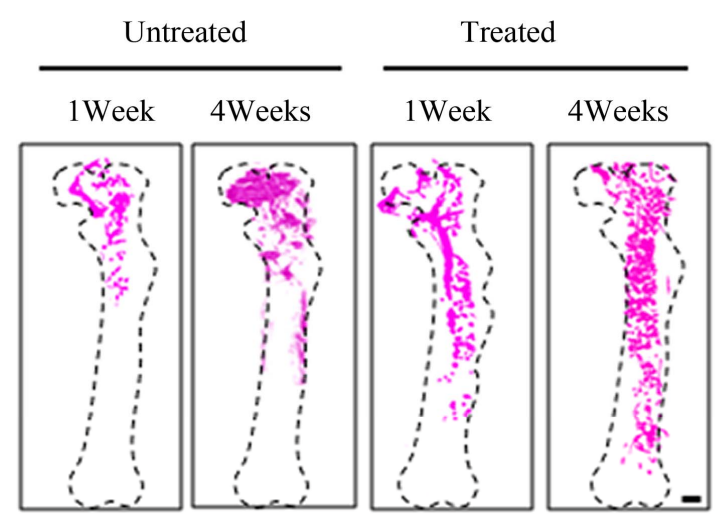

(a)

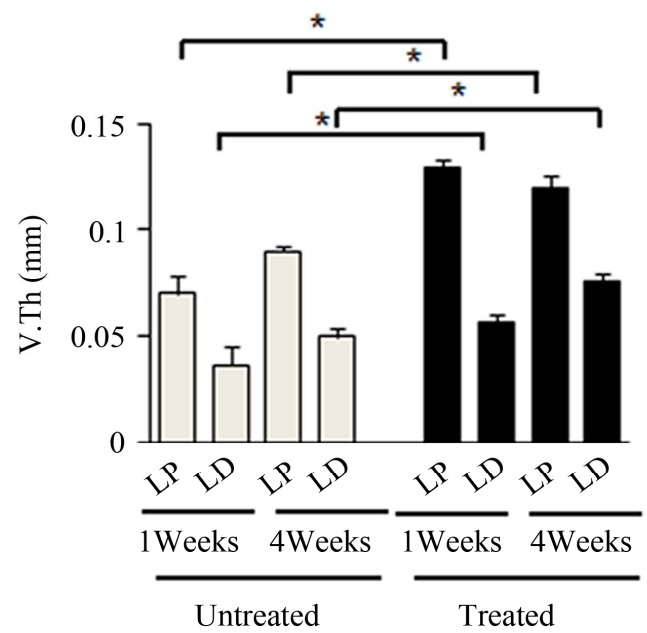

(c)

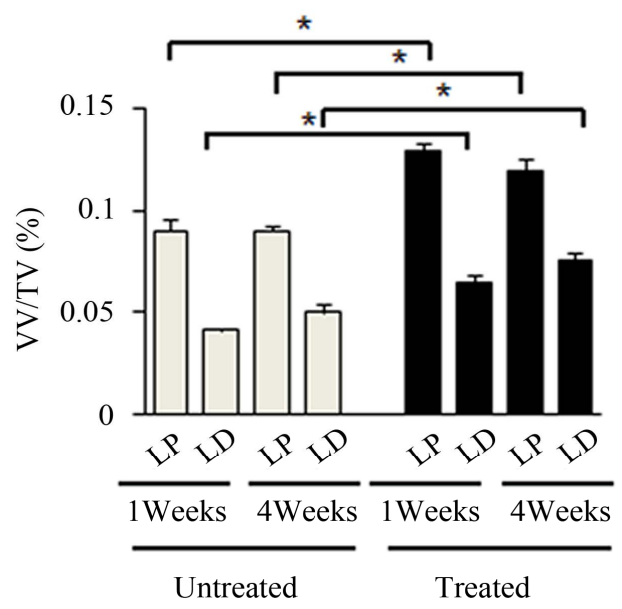

(b)

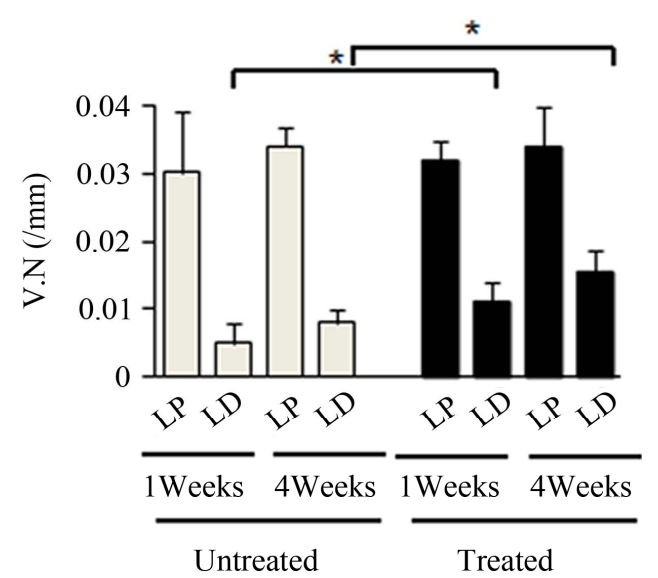

(d)

Figure 4. Effects of antioxidant on irradiation induced bone marrow angiogenesis. (a) Representative 3-dimensional $\mu \mathrm{CT}$ images of femora from the mice after local irradiation. Scale bar: $1 \mathrm{~mm}((\mathrm{~b})-(\mathrm{d})$ ); Quantitative $\mu \mathrm{CT}$ angiography analysis. Vascular volume fraction (VV/TV) (b); Vascular thickness (V.Th) (c); and Vascular number (V.N) (d) were measured. Data represent the mean $\pm \mathrm{SEM} ; \mathrm{n}=10 ;{ }^{*} p<0.05$. 
covery compared to their untreated counterparts in the left femur (Figures 4(b)-(d)). Vasculatures in the LD and LP antioxidant treated mice were more numerous, thicker, and had higher volume compared to the untreated group (Figures 4(b)-(d)). These data suggested that antioxidant administration is correlated with vasculature retention and recovery after irradiation.

\section{DISCUSSION}

Irradiation induces bone injury by producing free radicals that adversely affect the stem cell microenvironment for MSCs while damaging bone marrow blood vessels [1]. Improving bone marrow microenvironment and accelerating vasculogenesis could be therapeutically useful for patients to minimize radiation-induced bone injury. The aim of this study was to investigate the efficacy of antioxidant treatment in protecting bone marrow stem cells and promoting bone marrow vasculature recovery after radiation treatment.

Previous studies have shown that ascorbic acid acts as a protective agent against radiation and is involved in maintaining human fibroblast survival, DNA double stranded break repair, and sister chromatid exchanges [14]. Pretreatment with ascorbic acid may effectively prevent radiation-induced irreversible gastrointestinal damage (GI syndrome) by down-regulating apoptosis associated genes [15]. Improved survival as a result of a high antioxidant diet has been attributed to a reduction in radiation-induced oxidative stress and apoptosis of bone marrow hematopoetic stem cells [16]. The effects of antioxidant on MSCs survival and repopulation are still unclear. We found that histological analysis in ascorbic acid treated mice showed alleviated bone marrow microenvironment damage and substantial cellular repopulation 1 and 4 weeks after radiation. CFU-F and CFU-Ob assays indicated more active MSCs existing in both the LP and LD femurs in ascorbic acid treated mice. Our evidence suggests that administration of antioxidants reduced the effects of free radical damage and ROS, thus allowing better MSCs survival and recovery.

Several studies reported that MSCs reside in a perivascular niche, and bone marrow vasculature is a critical component of bone tissue and functions in maintenance of MSCs [17]. We found that high retention of the vasculature associating with antioxidant treatment is correlated with improved bone marrow recovery following irradiation. Reduction of free radical levels by ascorbic acid may contribute to the alleviation of vascular injury following irradiation. However, the mechanisms still need to be further investigated.

Our study suggests that administration of antioxidants prior to radiation exposure may initially limit the extent of radiation damage by allowing vasculature retention and higher MSCs survival. Such effects could therefore shorten the recovery time by allowing for earlier cellular repopulation of the radiated bone marrow. Previous studies have suggested that a diet high in antioxidants may improve patient prognosis following radiation treatments for cancer [18]. In this study, we provide suggestive evidence through in vivo analysis of the bone marrow itself. Furthermore, bone insufficiency could be correlated with bone marrow MSCs depletion from direct and indirect effects of radiation [1]. It could be proposed that retention of bone marrow MSCs provided by taking preventive antioxidants could function as a therapeutic approach to reducing the side effects on bone tissue following irradiation.

\section{ACKNOWLEDGEMENTS}

This project was supported by National Institutes of Health Grant AR 053973 (XC).

\section{REFERENCES}

[1] Cao, X., Wu, X., Frassica, D., Yu, B., Pang, L., Xian, L., Wan, M., Lei, W., Armour, M., Tryggestad, E., Wong, J., Wen, C.Y., Lu, W.W. and Frassica, F.J. (2011) Irradiation induces bone injury by damaging bone marrow microenvironment for stem cells. Proceedings of the National Academy of Sciences of USA, 108, 1609-1614.

doi:10.1073/pnas.1015350108

[2] Ng, A.K., Bernardo, M.V., Weller, E., Backstrand, K., Silver, B., Marcus, K.C., Tarbell, N.J., Stevenson, M.A., Friedberg, J.W. and Mauch, P.M. (2002) Second malignancy after Hodgkin disease treated with radiation therapy with or without chemotherapy: Long-term risks and risk factors. Blood, 100, 1989-1996. doi:10.1182/blood-2002-02-0634

[3] Taghian, A., de Vathaire, F., Terrier, P., Le, M., Auquier, A., Mouriesse, H., Grimaud, E., Sarrazin, D. and Tubiana, M. (1991) Long-term risk of sarcoma following radiation treatment for breast cancer. International Journal of Radiation Oncology, Biology and Physics, 21, 361-367. doi:10.1016/0360-3016(91)90783-Z

[4] Hopewell, J.W. (2003) Radiation-therapy effects on bone density. Medical and Pediatric Oncology, 41, 208-211. doi:10.1002/mpo.10338

[5] Baxter, N.N., Habermann, E.B., Tepper, J.E., Durham, S.B. and Virnig, B.A. (2005) Risk of pelvic fractures in older women following pelvic irradiation. The Journal of the American Medical Association, 294, 2587-2593. doi:10.1001/jama.294.20.2587

[6] Kwon, J.W., Huh, S.J., Yoon, Y.C., Choi, S.H., Jung, J.Y., Oh, D. and Choe, B.K. (2008) Pelvic bone complications after radiation therapy of uterine cervical cancer: Evaluation with MRI. American Journal of Roentgenology, 191, 987-994. doi:10.2214/AJR.07.3634

[7] Wernle, J.D., Damron, T.A., Allen, M.J. and Mann, K.A. (2010) Local irradiation alters bone morphology and increases bone fragility in a mouse model. Journal of Biomechanics, 43, 2738-2746.

doi:10.1016/j.jbiomech.2010.06.017 
[8] Clutton, S.M., Townsend, K.M., Walker, C., Ansell, J.D. and Wright, E.G. (1996) Radiation induced genomic instability and persisting oxidative stress in primary bone marrow cultures. Carcinogenesis, 17, 1633-1639. doi:10.1093/carcin/17.8.1633

[9] Down, J.D., Boudewijn, A., van Os, R., Thames, H.D. and Ploemacher, R.E. (1995) Variations in radiation sensitivity and repair among different hematopoietic stem cell subsets following fractionated irradiation. Blood, 86, 122127.

[10] Jaiswal, R.K., Jaiswal, N., Bruder, S.P., Mbalaviele, G., Marshak, D.R. and Pittenger, M.F. (2000) Adult human mesenchymal stem cell differentiation to the osteogenic or adipogenic lineage is regulated by mitogen-activated protein kinase. Journal of Biologic Chemistry, 275, 96459652. doi:10.1074/jbc.275.13.9645

[11] Senn, J.S. and McCulloch, E.A. (1970) Radiation sensitivity of human bone marrow cells measured by a cell culture method. Blood, 35, 56-60.

[12] Deng, H., Kennedy, C.W., Armour, E., Tryggestad, E., Ford, E., McNutt, T., Jiang, L. and Wong, J. (2007) The smallanimal radiation research platform (SARRP): Dosimetry of a focused lens system. Physics in Medicine and Biology, 52, 2729-2740. doi:10.1088/0031-9155/52/10/007

[13] Tang, Y., Wu, X., Lei, W., Pang, L., Wan, C., Shi, Z., Zhao, L., Nagy, T.R., Peng, X., Hu, J., Feng, X., Van Hul, W., Wan, M. and Cao, X. (2009) TGF-beta1-induced mi- gration of bone mesenchymal stem cells couples bone resorption with formation. Nature Medicine, 15, 757-765. doi:10.1038/nm.1979

[14] Fujii, Y., Kato, T.A., Ueno, A., Kubota, N., Fujimori, A. and Okayasu, R. (2010) Ascorbic acid gives different protective effects in human cells exposed to X-rays and heavy ions. Mutation Research, 699, 58-61.

[15] Yamamoto, T., Kinoshita, M., Shinomiya, N., Hiroi, S., Sugasawa, H., Matsushita, Y., Majima, T., Saitoh, D. and Seki, S. (2010) Pretreatment with ascorbic acid prevents lethal gastrointestinal syndrome in mice receiving a massive amount of radiation. Journal of Radiation Research (Tokyo), 51, 145-156. doi:10.1269/jrr.09078

[16] Wambi, C., Sanzari, J., Wan, X.S., Nuth, M., Davis, J., Ko, Y.H., Sayers, C.M., Baran, M., Ware, J.H. and Kennedy, A.R. (2008) Dietary antioxidants protect hematopoietic cells and improve animal survival after total-body irradiation. Radiation Research, 169, 384-396. doi:10.1667/RR1204.1

[17] Greenberger, J.S. and Epperly, M. (2009) Bone marrowderived stem cells and radiation response. Seminars in Radiation Oncology, 19, 133-139. doi:10.1016/j.semradonc.2008.11.006

[18] Brown, M.S., Buchanan, R.B. and Karran, S.J. (1980) Clinical observations on the effects of elemental diet supplementation during irradiation. Clinical Radiology, 31, 19-20. doi:10.1016/S0009-9260(80)80075-4 